\title{
Un travail qui ne compte pas? La valorisation monétaire du bénévolat associatif
}

Association volunteering and money value : is this activity extraneous to any type of measurement?

\section{Édith Archambault et Lionel Prouteau}

\section{OpenEdition}

\section{Journals}

Édition électronique

URL : http://journals.openedition.org/travailemploi/4799

DOI : 10.4000/travailemploi.4799

ISSN : $1775-416 \mathrm{X}$

Éditeur

DARES - Ministère du Travail

Édition imprimée

Date de publication : 15 décembre 2010

Pagination : $57-67$

ISSN : 0224-4365

Référence électronique

Édith Archambault et Lionel Prouteau, « Un travail qui ne compte pas ? La valorisation monétaire du bénévolat associatif », Travail et Emploi [En ligne], 124 | octobre-décembre 2010, mis en ligne le 15 décembre 2012, consulté le 30 avril 2019. URL : http://journals.openedition.org/travailemploi/4799 ; DOI : 10.4000/travailemploi.4799 


\title{
Un travail qui ne compte pas? La valorisation monétaire du bénévolat associatif
}

\author{
Édith Archambault (*) Lionel Prouteau (**)
}

\begin{abstract}
Le bénévolat est d'abord appréhendé comme un don de temps, or ce qui relève du don fait rarement bon ménage avec le calcul économique. Pourtant, le travail bénévole est aussi une importante ressource productive, qui compte pour les associations. Cet article se propose d'en déterminer la valeur monétaire. Dans un premier temps, sont examinées les difficultés que rencontre un tel exercice. Elles procèdent notamment du manque de régularité et de l'hétérogénéité des enquêtes réalisées sur le bénévolat. Elles tiennent aussi à la diversité des méthodes envisageables pour affecter une valeur monétaire à des activités qui, par définition, ne s'inscrivent pas dans cette métrique. Ces diffi aultés ne sont pourtant pas rédhibitoires dès lors que les statisticiens s'accordent sur des conventions partagées. Dans un second temps, l'article procède à une valorisation monétaire du bénévolat associatif français, à partir d'une enquête conduite auprès des associations par une équipe de recherche du Centre d'économie de la Sorbonne. Les estimations obtenues font apparaître que ce travail bénévole représente de $1 \%$ à près de $2 \%$ du PIB selon la méthode retenue, soit de $50 \%$ environ à $80 \%$ des salaires bruts versés par les associations à leurs salariés et, en tout état de cause, bien plus que les dons monétaires.
\end{abstract}

Le travail bénévole auprès des associations, s'il est d'abord appréhendé comme un don de temps, représente aussi une ressource productive importante pour ces dernières, dont le poids économique est progressivement mieux connu et, partant, reconnu. Les initiatives des acteurs associatifs ne sont pas étrangères à cette situation. Entre autres exemples, on peut évoquer la participation de certains d'entre eux à la constitution de listes communes aux employeurs de l'économie sociale à l'occasion des deux dernières élections prud'homales. Cette dynamique a connu un réel succès puisque ces listes ont obtenu en 2008 près de $20 \%$ des suffrages.

Mais cette meilleure connaissance du rôle productif des associations est également le fruit d'un effort de recherche croissant en sciences sociales depuis le début des années 1990. Cet effort a été puissamment stimulé par le programme comparatif

(*) Université de Paris 1 ; edith.Archambault@univ-paris1.fr (**) LEMNA, université de Nantes, BP 52231 Chemin de la Censive du Tertre, 44322 Nantes Cedex 3, France ; lionel. prouteau@univ-nantes.fr Les auteurs remercient Viviane Tchernonog pour les informations qu'elle leur a communiquées sur l'enquête 2005 du Centre d'économie de la Sorbonne auprès des associations. Ils remercient également deux rapporteurs anonymes pour leurs remarques et suggestions. Ils restent évidemment seuls responsables des erreurs qui demeureraient dans cet article.
Johns Hopkins sur le secteur sans but lucratif qui concerne à ce jour quarante-six pays. C'est dans le cadre de ce programme que des travaux approfondis ont été réalisés en France sur les associations, qui constituent la très grande majorité de ce secteur (Archambault, 1996). En lien avec ce programme international, le Centre d'études sur la société civile de l'université Johns Hopkins a œuvré conjointement avec la Division statistique des Nations unies à la rédaction d'un Manuel sur les institutions sans but lucratif dans le système de comptabilité nationale, publié en 2003. Ce manuel recommande la construction de comptes satellites des Institutions sans but lucratif (ISBL ci-après). À ce jour, trentedeux pays se sont engagés dans cette voie et une dizaine d'entre eux ont publié les leurs. En France, ce travail a été assuré par KAMINSKI (2005) à l'issue d'une mission confiée par l'INSEE. Au terme de son étude, la valeur ajoutée des ISBL est estimée à 2,9\% du PIB pour l'année 2002. Enfin, il est impossible d'évoquer les recherches récentes conduites sur les réalités associatives sans citer les travaux de Tchernonog $(2000,2007)$ effectués à partir de vastes enquêtes conduites auprès d'associations.

L'un des traits spécifiques des associations réside dans leur capacité à mobiliser un bénévolat qui est tout à la fois un facteur majeur de leur identité, puisque leur création est nécessairement le fruit d'engagements bénévoles, et une ressource productive non négligeable. Les recherches sur le secteur sans but lucratif ont donc assez logiquement engendré un 
intérêt croissant pour ces activités non rémunérées. Le présent article s'inscrit dans le cadre de cette attention portée au travail bénévole et se propose plus précisément d'en estimer la valeur monétaire. Cet exercice est utile notamment pour rendre le don de temps pleinement commensurable avec les autres types de ressources des associations et, par conséquent, pour en appréhender l'importance relative. Une comptabilité en termes exclusivement " physiques », en heures ou en emplois équivalents temps plein (ETP), ne permet pas de réaliser un tel examen comparatif. C'est pourquoi, parmi les trois versions des comptes des ISBL qu'il présente, le Manuel sur les institutions sans but lucratif dans le système de comptabilité nationale (UNITED NATIONS, 2003) en envisage un (la version c) qui affecte une valeur monétaire au travail bénévole et augmente donc à due concurrence la production et la valeur ajoutée des associations. Cette imputation monétaire n'est toutefois pas chose aisée et n'a d'ailleurs pas été tentée dans le compte satellite français. Elle soulève en effet un certain nombre de problèmes dont les principaux sont évoqués dans la première partie de cet article. Les difficultés rencontrées n'ont cependant pas un caractère rédhibitoire et devraient trouver des réponses harmonisées à l'échelle internationale grâce aux efforts réalisés sous l'égide de l'Organisation internationale du travail et du Centre pour les études sur la société civile de l'université Johns Hopkins. La seconde partie présente plusieurs estimations auxquelles donne lieu cette valorisation monétaire pour le bénévolat associatif français.

\section{Les questions posées par la valorisation monétaire du bénévolat}

Ces questions se situent à plusieurs niveaux. Le premier d'entre eux concerne la légitimité de cette opération de valorisation. En effet, en inscrivant le bénévolat dans l'ordre de la métrique monétaire, elle peut être suspectée d'en transformer profondément la nature pour n'y voir qu'un travail alors qu'il s'agit tout autant, sinon davantage, d'un don et d'un engagement. Imputer une valeur monétaire au bénévolat, ne serait-ce ce pas le réduire à son seul aspect économique de facteur de production en oubliant les autres registres sur lesquels il peut être décliné, notamment en tant que générateur de liens sociaux ou en tant que promoteur et défenseur de causes dans l'espace public ? Ainsi, l'un des participants à une table ronde organisée sur ce sujet par la revue $e$-Volunteerism considère-t-il cette opération comme étant pernicieuse (Keyboard Roundtable, 2003). Un tel point de vue illustre bien les tensions inhérentes aux situations critiques qui peuvent naître de la confrontation de formes différentes de justification (Boltanski, ThéVenot, 1991). Si cette question ne peut guère être approfondie dans les limites de cet article, il convient néanmoins d'observer que le fait de considérer le bénévolat comme un travail ne conduit pas inéluctablement à sa banalisation. Cela peut au contraire inciter à porter un regard sur le travail salarié moins sommaire que celui du modèle microéconomique standard de l'offre de travail, qui voit dans l'obtention d'un revenu l'unique finalité du salarié. «Penser le bénévolat comme travail » invite alors, en empruntant aux propos de SiMONETCusset (2004, p. 149), « à s'intéresser au travail comme à un engagement, en étudiant dans leur pluralité les modalités d'engagement dans le travail et les fondements de l'engagement au travail ». En d'autres termes, voir dans le bénévolat un travail, n'est-ce pas aussi suggérer qu'il peut y avoir du don dans le travail salarié (1) ? Quoi qu'il en soit, il n'est pas inutile de préciser que l'affectation d'une valeur monétaire au bénévolat ne saurait être la seule manière d'appréhender l'apport de ce comportement à la société. Il s'agit seulement de mieux documenter l'une de ses dimensions, celle qui relève de son rôle productif et qui contribue à la reconnaissance que lui portent les associations et plus largement la société. Il s'agit aussi d'admettre que l'activité économique ne saurait être circonscrite aux domaines du travail rémunéré. La remarque a son importance car elle conduit par exemple à considérer que, si les retraités sont bien des « inactifs » au sens du Bureau international du travail (BIT), ils n'en restent pas moins productifs dès lors qu'ils se consacrent à des activités bénévoles (Herzog, Morgan, 1992 ; Prouteau, WOLFF, 2007).

À un deuxième niveau, se pose le problème de la pertinence proprement économique de cette imputation monétaire, dans un cadre macro-économique qui est le nôtre ici et qui renvoie aux principes régissant la comptabilité nationale. VANOLI (2002, p. 307) rappelle que le SCN 93 se situe dans la continuité d'une acception large de la production économique définie comme activité dont « les sorties - biens ou services - peuvent être livrées ou fournies à d'autres unités, soit individuellement, soit collectivement, contre paiement [...] ou gratuitement ». Mais dans son cadre central, le SCN restreint le champ de la production à celui de l'emploi socialement organisé. VANOLI note à ce propos que si « le travail bénévole dans le cas d'associations constitue un cas limite », il n'est pas inclus pour autant dans le cadre central mais peut être pris en compte dans un compte satellite (ibid., p. 307). En prévoyant une valorisation monétaire du bénévolat dans la troisième version du compte satellite des ISBL, le Manuel des Nations unies est donc en cohérence avec cette orientation.

On sait, en revanche, que les comptables nationaux expriment fréquemment les plus vives réserves à l'égard des travaux visant à construire

(1) Ainsi, certains modèles de salaires d'efficience suggèrent que la relation salariale emprunte à la logique du don - contredon (AKERLOF, 1982). 
des agrégats élargis par rapport au PIB et pouvant apparaître comme alternatifs à ce dernier (GADREY, JANY-CATRICE, 2007). Le récent rapport de la Commission sur la mesure des performances économiques et du progrès social est de nature à relancer le débat sur l'opportunité de tels travaux. Il convient à ce propos de noter que ce rapport reste d'une grande discrétion sur le bénévolat alors qu'il évoque, dans son premier chapitre, la possibilité d'intégrer le travail domestique et même les loisirs dans la mesure d'un « revenu total » (STIGLITZ, SEN, Fitoussi, 2009) (2). Ce silence sur le travail bénévole associatif est d'autant plus surprenant qu'il est socialement organisé et probablement moins difficilement comparable à des substituts marchands que le travail domestique. D'ailleurs plusieurs indicateurs alternatifs de richesse ou de bien-être intègrent déjà une estimation monétaire du bénévolat. Tel est le cas du Genuine Progress Indicator ou indicateur de progrès véritable (TALBERT, CoBB, SlatTERY, 2007) et de l'Index of Economic Well-being (indice du bien-être économique) d'Osberg et SHARPe (1998), du moins dans l'application que ces auteurs en font pour le Canada.

Parmi les raisons expliquant leurs réticences à l'égard de nouveaux agrégats de richesse, les comptables nationaux mettent notamment en avant les incertitudes entourant la mesure des activités qu'il s'agirait d'intégrer. Évoquant la question des activités domestiques, VANOLI (2002, p. 362) écrit ainsi qu'elle « ne posent pas à la comptabilité nationale normalisée [...] de problème de principe quant à leur inclusion dans une notion élargie de la production et de la consommation, mais de sévères questions de mesure ». Le bénévolat n'échappe pas à cette remarque. Les difficultés que soulève sa mesure peuvent être appréhendées sous deux aspects : celui de la connaissance de son volume, exprimé en heures ou en emplois ETP, et celui de la conversion de ce volume en grandeurs monétaires.

\section{Mieux connaître le volume du travail bénévole}

Estimer le volume du bénévolat requiert la collecte de données par voie d'enquêtes, qu'elles soient spécifiques à ce comportement ou plus générales. Elles sont réalisées à l'initiative de chercheurs ou d'organisations privées qui se consacrent à la promotion du tiers secteur et du bénévolat, comme The Independent Sector aux États-Unis, ou encore par des instituts statistiques officiels. Leur régularité est néanmoins très inégale d'un pays à l'autre. En France, une seule enquête a été menée sur le sujet à ce jour par la statistique publique. Il s'agit de celle

(2) Dans ce rapport, les activités bénévoles sont curieusement très furtivement citées au titre de la production domestique (pp. 39 et 139) et ne sont véritablement envisagées que dans leur fonction de lien social (pp. 57 et 203). que l'INSEE a réalisée en octobre 2002 sur la vie associative au titre du volet variable de l'Enquête permanente sur les conditions de vie des ménages (EPCVM).

Comme ces enquêtes ne sont pas nécessairement similaires sous l'angle des méthodes qu'elles mettent en œuvre, la comparaison de leurs résultats est délicate, que ce soit au niveau international ou dans le cadre d'un même pays. Ainsi l'enquête australienne conduite pour l'année 2006 par le Bureau of Statistics conclut à un taux de participation au bénévolat de $34 \%$, alors que celle réalisée l'année précédente par le Department of Family and Community Services estime ce taux à $41 \%$. Aux États-Unis, l'U.S. Current Population Survey obtenait un taux de 28,8\% en 2003 alors que l'enquête de The Independent Sector parvenait à $44 \%$ pour l'année 2000. STATISTIQUE CANADA a connu une situation similaire avec les changements méthodologiques introduits dans l'enquête 2004 sur le don, le bénévolat et la participation : le taux de participation obtenu fut alors de $45 \%$ contre $27 \%$ pour l'enquête précédente portant sur l'année 2000 (STATISTIQUE Canada, 2006). Steinberg, Rooney et Chin (2002) se livrent à une analyse de sensibilité particulièrement intéressante, consistant à comparer les estimations en matière de bénévolat (taux de participation et durées qui lui sont consacrées) déduites d'enquêtes méthodologiquement distinctes, réalisées sur des échantillons de population de l'État américain de l'Indiana. L'exercice montre que les résultats peuvent différer dans des proportions très importantes. Les taux de participation varient de $29 \%$ à $74 \%$ et le volume horaire annuel moyen par bénévole d'un peu moins de 80 heures à environ 350 .

Cette instabilité des résultats relève de plusieurs facteurs. Le nombre et la formulation des questions ont une importance particulière. Interroge-t-on l'enquêté directement sur son bénévolat, en le laissant juge de la définition de ce comportement, ou bien son éventuelle participation est-elle inférée par l'enquêteur à partir de questions plus indirectes ? Le choix fait à ce niveau peut conduire à des différences non négligeables dans le dénombrement des bénévoles (Prouteau, WolfF, 2004a). La capacité des questionnaires à mobiliser les souvenirs du répondant, par l'inclusion éventuelle d'aide-mémoire concernant les activités ayant pu être réalisées au titre du don de temps, joue également un rôle important pour rendre compte des écarts constatés.

L'incertitude qui pèse sur le calcul du volume du bénévolat appelle donc un effort d'homogénéisation tant conceptuelle (comment définit-on le bénévolat ?) que méthodologique (comment doit-on concevoir les enquêtes à son sujet ?). Cet effort a été encouragé par une recommandation du BIT avalisée en décembre 2008 à Genève lors de la $18^{\mathrm{e}}$ conférence des statisticiens du travail. Il est ainsi préconisé d'adjoindre à l'enquête sur la force de travail, qui 
existe à peu près partout dans le monde et qui, en France, est l'enquête Emploi, un bref questionnaire sur le travail bénévole. Pour faciliter la mise en œuvre de cette recommandation, un manuel méthodologique et un questionnaire ont été élaborés dans le cadre d'un partenariat entre le BIT, l'organisation des Volontaires des Nations unies, la Division statistique des Nations unies et le Centre d'études de la société civile de l'université Johns Hopkins (3). Les perspectives ainsi ouvertes d'une systématisation et d'une harmonisation des enquêtes sur le bénévolat sont évidemment de nature à en faciliter la mesure. Mais cette dernière requiert également la résolution d'un deuxième problème relatif à l'imputation d'une valeur monétaire à des flux qui, par définition, en sont dépourvus.

\section{Imputer une valeur monétaire au travail non rémunéré}

Cette question a fait l'objet de nombreux travaux portant principalement sur le travail domestique (Hawrylyshyn, 1976 ; Chadeaun, Fouquet, 1981 ; Chadeau, 1985, Goldschmidt-Clermont, 1993) ou l'aide informelle (VAN DEN BERG, BROUWER, Koopmanschap, 2004 ; Davin, Paraponaris, Verger, 2009) mais qui concernent tout aussi bien le bénévolat associatif. Plusieurs méthodes sont a priori envisageables pour mener à bien cette valorisation monétaire.

La méthode de l'output consiste à valoriser les services réalisés grâce au bénévolat en leur affectant les prix auxquels leurs homologues sont offerts dans un cadre marchand. Il convient ensuite de défalquer le coût des consommations intermédiaires acquises pour assurer ces services, la rémunération d'éventuels salariés ayant contribué eux aussi à cette production ainsi que la consommation de capital fixe, pour obtenir in fine la valeur ajoutée par le travail bénévole. Cette méthode est d'un point de vue théorique la plus satisfaisante (GOLDSCHMIDTClermont, 1993), mais elle soulève des difficultés de mise en œuvre puisqu'elle suppose l'existence de substituts marchands aux services bénévoles, ce qui est loin d'être toujours le cas. Même lorsqu'ils existent, l'affectation des prix de ces substituts aux services bénévoles peut être discutée si l'on considère que ces prix ne traduisent pas nécessairement ce que serait la disposition à payer des bénéficiaires de ces services. BROWN (1999) prend ainsi l'exemple de prestations bénévoles en direction de

(3) La version provisoire de ce manuel et de ce questionnaire est consultable sur le site du Centre d'études de la société civile de l'université Johns Hopkins : http://www.ccss.jhu. edu/index.php ? section $=$ content $\&$ view $=9 \&$ sub $=12 \&$ tri $=106$. Archambault et Prouteau (2009) présentent et discutent en détail le questionnaire dont la rédaction finale a été adoptée par le BIT le 12 octobre 2010 avec quelques modifications mineures dont la plus importante vise à clarifier la distinction entre formation et bénévolat. populations défavorisées et soutient que la valeur que ces dernières leur attribuent peut être inférieure à leur valeur de marché. Pour appréhender la véritable disposition à payer des bénéficiaires, l'auteur recommande alors le recours à une méthode de la variation équivalente, laquelle engendre des difficultés d'un autre ordre.

S'il est difficilement envisageable de la généraliser dans le cadre d'une valorisation macroéconomique du bénévolat, cette méthode de l'output est néanmoins susceptible d'apporter des éclairages intéressants à un niveau plus désagrégé. Ainsi Hourigan (2001) évalue-t-elle sur cette base la valeur de la contribution bénévole d'un service australien de lutte contre les incendies et de secours d'urgence, en estimant le montant que la collectivité serait disposée à payer pour obtenir des services similaires en l'absence de bénévolat.

L'imputation d'une valeur monétaire au travail bénévole peut alternativement être conduite en retenant l'optique des coûts de production qui consiste à affecter au temps donné un salaire implicite. Il s'agit de la méthode dite des inputs. Se pose alors la question du choix du salaire de référence. Plusieurs approches sont possibles. Celle des coûts d'opportunité opte pour la rémunération que le bénévole pourrait obtenir si, au lieu d'être consacré gratuitement à une association, son temps était affecté à son activité professionnelle. Cette approche pose de nombreux problèmes, notamment lorsqu'il s'agit de traiter le cas des bénévoles inactifs. La difficulté ne peut que très partiellement être surmontée par le calcul d'un salaire potentiel, car ce dernier n'a de sens que dans des situations où l'individu est en capacité d'arbitrer entre le travail et ses autres activités : ce n'est le cas ni chez ceux pour qui l'inactivité est subie, ni chez les retraités (puisque le départ en retraite est très souvent contraint) (4). Outre les difficultés «techniques » auxquelles elle doit faire face, la méthode des coûts d'opportunité conduit à valoriser différemment de mêmes activités du fait que les bénévoles qui les réalisent perçoivent des rémunérations professionnelles distinctes. Faire ce choix serait donc clairement inadéquat pour une valorisation effectuée dans l'optique d'un compte satellite des ISBL s'efforçant d'appréhender l'importance que représente le bénévolat du point de vue des organisations qui mobilisent cette ressource productive. À cette fin, l'approche dite des coûts de remplacement est plus appropriée. Elle consiste à calculer le coût que représenterait, pour les associations, la substitution d'un travail rémunéré au travail bénévole. Cette hypothèse de remplacement a un pur statut méthodologique et ne sous-entend nullement que cette substitution salariés-bénévoles

(4) Lorsque cette méthode de valorisation est mise en œuvre, l'estimation est souvent faite sur la seule population en âge d'activité (Prouteau, WolfF, 2004 ; Pho, 2004). 
pourrait être mise en œuvre systématiquement (5). En pratique, beaucoup d'associations ne seraient pas capables, faute de moyens, de compenser la perte de leurs bénévoles et, quand bien même elles le seraient, le remplacement ne pourrait concerner les administrateurs, puisqu' un conseil d'administration composé de salariés serait incompatible avec le statut non lucratif et la gestion désintéressée.

La méthode des coûts de remplacement fait également l'hypothèse implicite que l'efficacité productive du bénévole est en moyenne identique à celle d'un salarié affecté à la même tâche. Cette similitude postulée des productivités bénévole et salariée peut être contestée au motif que la « convention de bénévolat » n'est pas du même ordre que le contrat de travail et qu'il peut être difficile pour les associations d'obtenir de certains de leurs bénévoles des contributions du type et de la qualité souhaités. L'existence de situations dans lesquelles le bénévole représente un apport très limité ou nul, voire négatif, pour son association, n'est guère contestable. Mais, d'un autre côté, certaines tâches sont susceptibles d'être effectuées de manière plus satisfaisante lorsqu'elles sont confiées à un bénévole plutôt qu'à un salarié : c'est le cas notamment quand elles ont une forte intensité relationnelle et que leur réalisation à titre d'activités rémunérées peut introduire chez le bénéficiaire du service un soupçon sur les intentions et les dispositions du prestataire à son égard (GoRz, 1988). Ce peut être également le cas lorsque les activités exigent un fort degré d'engagement, requérant de la part de ceux qui les réalisent de solides convictions. De telles situations ne sont-elles pas alors susceptibles de susciter le doute sur l'hypothèse d'une substitution du salariat au bénévolat? Une telle hypothèse relève en effet du critère de la tierce personne (HAWRYLYSHYN, 1977) qui conduit à définir le travail comme une activité pouvant être effectuée par un tiers sans que son résultat en soit affecté. Si ce critère permet de distinguer le travail du loisir, et le bénévolat de la participation associative en tant que pur usager des services réalisés, n'est-il pas plus problématique pour identifier la nature d'activités à forte intensité militante par exemple ? Cette interrogation est légitime. Elle rappelle que l'exercice auquel se prête cet article n'est certainement pas sans limite. Néanmoins, on observera que le bénévolat n'a pas le monopole de l'engagement militant. Ce dernier peut en maintes circonstances animer tout aussi bien les travailleurs rémunérés, qu'ils soient, entre autres exemples, permanents d'organisations syndicales

(5) La méthode du coût d'opportunité s'inscrit dans une approche en termes de «manque à gagner » et relève des modèles microéconomiques d'allocation du temps, tandis que la méthode du coût de remplacement procède plutôt d'une analyse en termes de « manque à dépenser » dans le cadre d'un modèle de consommation (CAILLAVET, 1998). ou salariés d'associations de défense de droits et de causes.

L'approche en termes de coûts de remplacement présente deux variantes. Celle dite du spécialiste (ou du substitut spécialisé) consiste à imputer à chaque tâche bénévole la rémunération que percevrait un spécialiste de la tâche en question. Cette variante requiert une information très fine et à ce jour rarement disponible sur les activités bénévoles et sur le niveau de qualification qu'elles impliquent. La deuxième variante retient comme rémunération implicite celle perçue par un salarié généraliste qui réaliserait ainsi l'ensemble des activités affectées au travailleur bénévole. Il reste alors à s'accorder sur cette rémunération. Le Manuel sur les institutions sans but lucratif dans le système de comptabilité nationale, conscient de la difficulté de mettre en œuvre la variante du substitut spécialisé, propose de choisir comme salaire de référence celui qui est versé dans la catégorie professionnelle des services communautaires et sociaux.

En pratique, les évaluations qui ont été réalisées dans différents pays optent à de très rares exceptions près (6) pour la méthode du coût de remplacement, mais les salaires servant de références présentent une grande variabilité. Si le compte satellite canadien opte pour le salaire des professions des services sociaux, le programme Johns Hopkins, dans l'étude comparative qu'il a menée sur trente-cinq pays, retient le salaire moyen du domaine d'activité dans lequel l'activité bénévole se déroule. Le compte satellite néo-zélandais des institutions sans but lucratif calcule, pour sa part, un taux de salaire de référence qui se présente comme une moyenne pondérée des salaires professionnels correspondant aux activités assurées par les bénévoles, les coefficients de pondération étant obtenus à partir des volumes horaires consacrés à chacune des activités en question (statistics New Zealand, 2007). Ce choix incline donc plutôt vers la variante du substitut spécialisé. Le compte satellite australien des institutions sans but lucratif emprunte une démarche assez similaire, mais il pondère les salaires professionnels de référence par le nombre de bénévoles engagés dans chacune des activités considérées, faute de disposer des volumes horaires concernés (Australian Bureau of Statistics, 2002). Dans d'autres travaux, le salaire imputé est plus simplement le salaire moyen calculé au niveau national. Tel est le choix fait par Low, Butt, Ellis Paine et David SMith (2007) dans leur étude sur le bénévolat britannique. Cette façon de procéder est toutefois problématique, puisqu'elle conduit à intégrer, dans

(6) Ainsi Ironmonger (2009) retient la méthode du coût d'opportunité.L'Australian BuREAU OFS TATISTICS (2000)présente une estimation monétaire du travail non rémunéré sur la base du coût d'opportunité et du coût de remplacement, tout comme PHO (2004) pour les États-Unis. 
le calcul de la rémunération implicite du bénévolat, les salaires relatifs à des métiers et versés dans des secteurs d'activités largement étrangers au monde associatif.

Une autre question ressortit à ce qu'il faut entendre par salaire. Dans l'optique du coût d'opportunité, il s'agira du salaire net de cotisations et d'impôts, qui est celui censé présider aux arbitrages individuels entre les activités professionnelles et les autres activités. La logique du coût de remplacement conduira en revanche à retenir comme salaire implicite celui qui traduit le coût du travail tel qu'il est appréhendé par l'employeur (Schmid, SouzA-Posa, WIDMER, 2002). Par conséquent, il s'agira du salaire superbrut, c'est-à-dire cotisations sociales des salariés et des employeurs incluses. En cohérence avec l'hypothèse de substitution, il faudra y ajouter les congés payés.

\section{Une application au bénévolat associatif français}

En France, quelques travaux se sont efforcés, au moins à titre exploratoire, de valoriser monétairement le travail bénévole réalisé en organisations, que celles-ci aient ou non le statut d'association. Tous mettent en œuvre la méthode des coûts de remplacement. Ainsi Archambault (1996) parvient à une estimation de 14,4 milliards d'euros pour l'année 1990, soit $1,13 \%$ du PIB. Le salaire implicite est celui en vigueur dans les domaines d'activités qui sont ceux des organismes accueillant les bénévoles. L'exercice fut réédité ultérieurement pour l'année 1995 (ARCHAMBAUlt, 2002). Le résultat obtenu est alors nettement plus élevé (30,5 milliards d'euros soit $2,55 \%$ du PIB), mais le volume du travail bénévole y est probablement surestimé car l'enquête qui a servi de support à cette mesure ne permet pas de distinguer bénévolat régulier et bénévolat occasionnel. Prouteau et WolfF (2004b) se livrent au même type d'opération à partir de l'enquête Vie associative conduite par l'INSEE en 2002 auprès des ménages. Leurs estimations varient selon le salaire implicite choisi pour l'imputation. La ressource bénévole représente $0,76 \%$ du PIB $(0,67 \%$ pour le seul bénévolat associatif) lorsque le salaire de référence est le SMIC, mais $1,10 \%(0,97 \%$ pour le bénévolat associatif) quand il s'agit du salaire moyen versé par les associations du domaine d'activité concerné.

\section{Le recours à une enquête auprès d'associations}

L'investigation dont les résultats sont ici communiqués présente l'originalité d'avoir été réalisée sur des données collectées auprès d'associations et non auprès de ménages, comme c'est le cas dans les travaux antérieurs aussi bien en France qu'au niveau international. Les deux sources d'informations ont leur intérêt mais aussi leurs limites. Outre la connaissance des caractéristiques sociodémographiques des bénévoles qu'elle est la seule à autoriser, l'enquête auprès des ménages permet d'informer sur le type de tâches que les répondants réalisent dans le cadre de leurs activités bénévoles et sur les durées qu'ils y consacrent. On ne peut exclure que ces durées soient surestimées s'agissant d'un comportement qui bénéficie d'une forte légitimité sociale ( $\mathrm{PHO}$, 2004, p. 16). Mais le principal problème est relatif à la connaissance parfois sommaire qu'ont les répondants des organismes auxquels ils donnent du temps, notamment en ce qui concerne leur nature juridique. Cette situation peut faire obstacle au repérage précis du bénévolat associatif, le seul à être concerné par le compte satellite des ISBL (7).

Dans les enquêtes auprès des organisations, ce problème est d'emblée résolu puisque l'enquêteur choisit lui-même la nature des structures enquêtées. Mais la difficulté naît dans ce cas de la connaissance très relative qu'ont ces organisations du volume $\mathrm{du}$ bénévolat dont elles font usage. En effet, l'enregistrement régulier de ces flux de ressources bénévoles n'est pas systématique (United NATIONS, 2003, p. 270 ; МooK, Sousa, Elgie, Quarter, 2005). De plus, même lorsqu'elles les comptabilisent, les associations peuvent avoir tendance à les sous estimer du fait qu'elles omettent parfois d'y inclure le bénévolat de leurs administrateurs (MERTENs, LeFÈBVRE, 2002).

Puisqu'aucune des deux sources (individus ou associations) ne se révèle indiscutablement optimale pour recueillir les données sur le travail bénévole, il convient de recourir aux deux types d'enquêtes pour pouvoir confronter les estimations auxquelles elles conduisent. Mais si les enquêtes réalisées en direction des ménages sont peu fréquentes et peu régulières, particulièrement en France, celles menées auprès des associations sont encore plus rares. En dépit des recommandations du Conseil national de l'information statistique (CNIS) (NEYRET, Nivlet, Rault, 1998) et des souhaits exprimés dans plusieurs rapports parlementaires récents (LANGLAIS, 2008 ; MoRAnGe, 2008), le monde associatif en tant que tel demeure largement ignoré par la statistique publique. Dans ce contexte, les données collectées au moyen d'enquêtes auprès d'associations par une équipe de recherche du Centre d'économie de la Sorbonne animée par Viviane TCHERNONOG s'avèrent particulièrement précieuses. La dernière de ces enquêtes a été réalisée en 2005 (TCHERNONOG, 2007). C'est elle qui est ici utilisée ( $c f$. encadré 1).
(7) Prouteau et Wolff (2004b) estiment que le bénévolat organisé (également appelé formel) destiné à des structures qui ne sont pas des associations représente de 11 à $12 \%$ du total. Ces structures peuvent être notamment des organismes publics (par exemple des écoles faisant appel au bénévolat parental pour l'accompagnement des sorties scolaires...). 
Encadré 1

\section{L'enquête 2005-2006 du Centre d'économie de la Sorbonne auprès des associations}

L'enquête a été réalisée par voie postale. Les questionnaires ont été adressés aux associations via les mairies, qui ont pu ainsi faire bénéficier les chercheurs de leur connaissance du tissu associatif local. 1725 communes ont accepté de collaborer à l'enquête. 13000 questionnaires ont été renseignés mais, certains l'étant trop incomplètement, l'échantillon final contient 9265 associations. Pour réaliser les extrapolations à la France entière l'échantillon a été redressé à partir des données relatives aux associations employeurs du fichier SIRENE.

Les données collectées concernent différents aspects de la vie des associations. Elles permettent notamment d'identifier leur domaine d'activité, leur ancienneté, mais aussi de connaître leurs budgets, leurs modes de financement, leur emploi salarié, leur gouvernance et leurs logiques d'action. Elles donnent également des informations sur le bénévolat (nombre de bénévoles et volumes horaires reçus à ce titre). Près d'une association sur deux n'a pas répondu aux questions sur les durées du travail bénévole, ce qui témoigne de leur connaissance très imparfaite de leurs ressources en travail non rémunéré. Les réponses communiquées permettent par extrapolation d'estimer à plus d'un milliard et demi le nombre annuel d'heures ainsi données en 2005, ce qui représente 935400 emplois en équivalent temps plein (ETP), le calcul étant fait sur la durée effective d'un temps plein. Un peu plus des trois quarts du travail bénévole est assuré dans des associations sans salarié, le petit quart restant l'étant dans des associations employeurs. Pour une présentation exhaustive de cette enquête, se reporter à TCHERNONOG (2007).

\section{Les ressources bénévoles en associations : de 1 à près de $2 \%$ du PIB}

La méthode retenue dans cet article est celle du coût de remplacement dans sa version du généraliste. En effet, il n'est pas possible à partir de l'enquête de Centre d'économie de la Sorbonne de distinguer les différentes tâches assurées par les bénévoles et, partant, de mettre en œuvre la variante du substitut spécialisé. Les ressources bénévoles sont exprimées en emplois ETP calculés sur la base de la durée légale (et non effective) de travail, c'està-dire 1607 heures par an. Le volume du bénévolat est ainsi estimé à hauteur de 957000 ETP, soit un niveau légèrement supérieur à celui qu'obtient TCHERNONOG sur la base d'une durée annuelle du travail un peu plus élevée.

Le calcul de la rémunération implicite imputée à un ETP bénévole annuel se fait en plusieurs étapes. La première consiste à identifier un salaire horaire brut moyen de référence à partir des déclarations annuelles de données sociales (DADS) de l'année

\section{Encadré 2 \\ Le calcul des cotisations sociales des employeurs}

Les DADS permettent de connaître les salaires bruts perçus par les salariés. Mais dans l'optique d'une valorisation monétaire du bénévolat au coût de remplacement, il importe d'estimer le coût salarial total que les associations devraient supporter si elles substituaient du travail rémunéré à leur bénévolat. Il faut donc inclure dans ce coût les cotisations sociales à la charge des employeurs. Parce que le taux de ces cotisations varie en fonction du salaire, se pose la question du taux pertinent pour la rémunération de référence.

Dans la variante qui considère le SMIC comme salaire implicite, le taux de cotisation retenu est celui en vigueur pour ce niveau de salaire en 2005, soit 19,04\%. Dans la seconde variante (salaire moyen dans l'action sociale), il aurait été envisageable de choisir le taux correspondant en 2005 à ce salaire moyen (soit $42,15 \%$ pour un salaire mensuel de 1865,54 euros) mais cette manière de procéder revenait à faire fi de la distribution des salaires dans le domaine d'activité considéré. Il a été jugé préférable d'opter pour un taux moyen pondéré obtenu de la manière suivante. Les salaires bruts mensuels moyens des différentes catégories socioprofessionnelles simplifiées (cadres et professions intellectuelles, professions intermédiaires, employés, ouvriers qualifiés et ouvriers non qualifiés) ont d'abord été estimés. Puis, à partir de la grille des cotisations des employeurs en vigueur en 2005 , les taux applicables à ces salaires moyens ont été déterminés. Chacun de ces taux s'est vu affecter un coefficient de pondération calculé en rapportant le volume horaire de la catégorie socioprofessionnelle concernée au volume horaire total de travail relatif au poste Q22 de la NES. La somme de ces taux pondérés donne le taux finalement appliqué, soit $37,06 \%$.

$S$ 'agissant de la troisième variante qui prend pour référence le salaire moyen du code $913 \mathrm{E}$ de la NAF rev. 1, il n'était pas possible d'opérer selon la démarche précédente faute de connaître la répartition du volume de travail par catégorie socioprofessionnelle. A été retenu le ratio des charges sociales aux salaires bruts tel qu'il apparaît dans le compte satellite des ISBL (KAMINSKI, 2005), soit $39,1 \%$. Certes, le périmètre de ce compte ne se limite pas strictement aux organismes à statut associatif et les plus petits organismes employeurs (moins d'un salarié ETP sur l'année) en ont été exclus. De plus, ce compte satellite porte sur l'année 2002, antérieure à la « réduction Fillon » qui a pris en 2005 le relais des dispositifs antérieurs d'allégement de charges. Toutefois, le rapport de la Commission des comptes de la Sécurité sociale de 2006 observe qu'en ce qui concerne l'année 2005, la montée en charge du nouveau dispositif ne s'est pas faite à plein régime et que la croissance du montant des exonérations est restée modérée. Aussi le taux ici retenu paraît constituer une approximation raisonnable. 
2005 puis à calculer un revenu mensuel en effectuant le produit de ce salaire horaire par 151,67 (soit 35 x 52 / 12). À ce revenu mensuel brut sont ensuite ajoutées les cotisations sociales des employeurs. L'encadré 2 précise les différentes manières dont les taux de ces cotisations ont été déterminés. À l'issue de cette seconde étape, il est aisé d'obtenir le coût salarial annuel total tel qu'il est appréhendé par l'employeur. Il reste alors à multiplier ce coût par le volume du bénévolat exprimé en emplois ETP pour en obtenir une valeur monétaire agrégée. Celle-ci est rapportée au PIB élargi de l'année 2005 afin d'obtenir un indicateur plus suggestif de l'importance cette ressource (8).

Trois variantes de valorisation ont été considérées. Elles diffèrent entre elles par le salaire de référence choisi. La première variante opte pour le SMIC, à savoir 8,03 euros l'heure pour l'année 2005. Cette variante sert de seuil minimum à l'opération de mesure ici réalisée. Elle peut traduire deux hypothèses distinctes : ou bien les bénévoles sont supposés être en général moins efficaces que les salariés dans la réalisation de leurs tâches ; ou bien les tâches affectées aux bénévoles sont considérées être moins qualifiées que celles qui sont attribuées à la main-d'œuvre rémunérée. Il n'est pas inutile de remarquer que les associations, très peu nombreuses, qui pratiquent la valorisation de leur bénévolat (notamment dans le cadre de leurs relations avec leurs financeurs) le font parfois en se référant au SMIC (Bevant, Noguès, Prouteau, 2001).

La deuxième variante suit la préconisation du Manuel sur les institutions sans but lucratif dans le système de comptabilité nationale. Elle retient donc le salaire horaire brut moyen dans les services d'action sociale, tel qu'il apparaît au poste Q22 de la Nomenclature économique de synthèse (NES niveau 3) qui était associée jusqu'en 2007 à la Nomenclature des activités française (NAF) rev. 1 (9). Ce salaire s'élevait en 2005 à 12,30 euros.

La troisième variante est basée sur la rémunération versée par les associations. Le salaire horaire brut retenu est alors celui relatif au code $913 \mathrm{E}$ de la NAF rev. 1, «associations non classées par ailleurs », à savoir 13,90 euros. Ce choix a été préféré à celui du salaire du poste R21 de la NES qui concerne les « activités associatives ». Retenir ce dernier eût en effet exposé au risque d'une surestimation du fait de l'inclusion dans ce poste des organisations patronales et consulaires, des organisations professionnelles et des organisations politiques dont les salaires moyens sont substantiellement supérieurs à celui du code 913E.

(8) Le PIB élargi est calculé en ajoutant au PIB tel qu'il est obtenu en comptabilité nationale la valeur imputée au bénévolat. (9) La NAF rev. 1 a cédé la place en 2008 à la NAF rev. 2.
Le tableau 1 présente les estimations ainsi obtenues. La valeur monétaire des ressources bénévoles varie du simple au double selon la variante retenue, représentant de 1 à près de $2 \%$ du PIB élargi. Il serait intéressant de comparer ces estimations à la masse salariale totale (cotisations sociales employeurs comprises) versée par les associations. Faute de données le permettant, un rapprochement a été opéré entre, d'une part, le montant des rémunérations brutes des salariés associatifs tels qu'ils sont communiqués dans une étude du CERPHI (2006) pour l'année 2005 à partir des données de l'ACOSS et, d'autre part, les résultats ici présentés dont ont été soustraites les cotisations sociales des employeurs. Les salaires bruts versés par les associations s'élèvent à 29,4 Mds d'euros alors que ceux imputés au bénévolat sont de 14 Mds d'euros dans la première variante, de 21,4 Mds dans la seconde et de 24,2 Mds dans la troisième.

Tableau 1 : La mesure du bénévolat associatif en 2005

\begin{tabular}{|l|c|c|c|}
\hline & \multicolumn{3}{|c|}{ Variantes relatives au salaire de référence } \\
\hline & SMIC & $\begin{array}{c}\text { Salaire action } \\
\text { sociale }\end{array}$ & $\begin{array}{c}\text { Salaire associatif } \\
\text { code NAF 913E }\end{array}$ \\
\hline $\begin{array}{l}\text { Coût salarial } \\
\text { annuel de réfé- } \\
\text { rence pour un } \\
\text { ETP }\end{array}$ & $17397 €$ & $30683 €$ & $35190 €$ \\
\hline $\begin{array}{l}\text { Valeur monétaire } \\
\text { imputée totale } \\
\text { dont bénévolat } \\
\text { en associations } \\
\text { employeurs }\end{array}$ & $16,7 \mathrm{Mds} €$ & $29,4 \mathrm{Mds} €$ & $33,7 \mathrm{Mds} €$ \\
\hline $\begin{array}{l}\text { Proportion du } \\
\text { PIB élargi }\end{array}$ & $0,96 \%$ & $7 \mathrm{Mds} €$ & $8 \mathrm{Mds} €$ \\
\hline
\end{tabular}

Source : calculs des auteurs à partir de l'Enquête du Centre d'économie de la Sorbonne auprès des associations 2005-2006.

\section{Éléments de discussion}

Les résultats ainsi obtenus peuvent susciter plusieurs remarques. La première relève de leur confrontation avec les estimations, rappelées plus haut, de Prouteau et WolfF (2004b) à partir de l'enquête Vie associative 2002 de l'INSEE. La comparaison n'a de pertinence que si elle est effectuée pour une variante identique. La seule disponible est celle qui retient le SMIC comme base de valorisation. Le résultat figurant dans le tableau 1 apparaît alors supérieur d'environ $25 \%$ à celui des auteurs susmentionnés. Comment expliquer cet écart ? On peut en voir une cause dans le fait que ces auteurs n'intègrent pas les congés payés dans le salaire de référence. Mais la source la plus importante de l'écart tient au volume du travail bénévole considéré : 720000 emplois ETP à partir de l'enquête de l'INSEE contre environ 950000 dans l'enquête du Centre d'économie de la Sorbonne. Eu égard à la période très courte qui sépare les deux enquêtes (trois ans), il est difficile d'imputer cette différence conséquente à un accroissement de l'engagement 
bénévole. C'est donc dans la nature distincte de ces enquêtes qu'il faut en trouver l'origine (celle de l'INSEE étant menée auprès des ménages, la seconde auprès des associations) sans pour autant que l'on puisse identifier la raison précise de l'écart constaté. Il est souhaitable que de futures enquêtes permettent d'éclairer cette question qui reste pour l'instant en suspens.

La deuxième remarque procède du rapprochement que l'on peut être tenté de faire entre l'apport que représente le bénévolat, d'une part, et celui que constituent les dons monétaires, d'autre part. L'exercice n'est pas aisé car la connaissance de ces dons reste approximative. Ils peuvent être estimés à partir de données fiscales, même s'ils sont alors probablement minorés du fait que les dons éligibles aux crédits d'impôts ne sont pas systématiquement déclarés et parce que les dons manuels, d'un montant le plus souvent très modeste, échappent à ce mode de comptabilisation. À partir de cette source fiscale, FACK et LANDAIS (2009) estiment à un peu plus de 1,4 milliard d'euros les dons monétaires réalisés en 2005. Utilisant les réponses collectées par l'enquête INSEE, PROUTEAU et WolfF (2004b) situent quant à eux la valeur de ces dons dans un intervalle compris entre 1,3 et 1,8 milliard d'euros en 2002. Il est donc clair que l'apport des bénévoles surpasse de très loin celui effectué en argent par les donateurs.

La troisième et dernière remarque est relative à la comparaison internationale des contributions bénévoles. Les investigations réalisées à l'étranger parviennent à des résultats variables. À titre d'exemple, le compte satellite de Nouvelle-Zélande estime que le bénévolat au profit des institutions sans but lucratif représente 2,3\% du PIB en 2004 (STATIStics New Zealand, 2007). En Australie, ce taux est de $1,4 \%$ pour 2000 (AustralianBureau ofSTATISTICS, 2002), identique à celui du Canada la même année (Statistique Canada, 2008). Рho (2004) obtient un taux de 1,26\% pour les États-Unis en 2001-2002. Mais, eu égard à la diversité des approches mises en œuvre dans ces travaux, il serait hasardeux de tirer des conclusions hâtives de la confrontation de ces résultats entre eux, ainsi qu'avec les estimations communiquées dans le présent article.

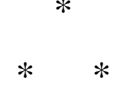

De telles comparaisons, comme d'ailleurs celles qui pourraient être réalisées dans le temps pour appréhender l'évolution de l'apport des bénévoles aux associations dans un même pays, ne manquent pas d'intérêt, dès lors qu'elles portent sur des données collectées sur la base d'enquêtes similaires et exploitées selon une même méthodologie. Une telle perspective suppose l'adoption de conventions statistiques permettant d'homogénéiser la définition du bénévolat et les opérations de valorisation monétaire le concernant. Ces conventions ne paraissent pas hors d'atteinte dès lors qu'est reconnue la nécessité de faire pénétrer le travail bénévole dans le périmètre de la production de richesses. À cet égard, il n'est pas incongru de partager le sentiment exprimé par GADREY (2006) à propos des conventions d'imputation d'une valeur monétaire au travail domestique mais qui vaut tout aussi bien et peut-être même mieux pour le bénévolat organisé : l'élaboration de telles conventions ne représente pas un défi plus grand que cela n'a été le cas pour les services d'intermédiation financière. Le manuel sur la mesure du bénévolat (Manual on the Measurement of Volunteer Work), dont le contenu a été récemment adopté par le BIT et qui préconise d'ajouter aux enquêtes nationales sur la force de travail un volet consacré au bénévolat, représente un pas important dans cette voie. 


\section{Bibliographie}

Akerlof G.A. (1982), "Labor Contract as Partial Gift Exchange", Quarterly Journal of Economics, Vol. 97, pp. 543-569.

Archambault E. (1996), Le secteur sans but lucratif. Associations et Fondations en France, Paris, Economica.

Archambault E. (2002), « Le travail bénévole en France et en Europe. Résultats du programme de recherche de l'université Johns Hopkins de comparaison internationale du secteur sans but lucratif », Revue française des affaires sociales, no 4, pp. 13-36.

Archambault E., Prouteau L. (2009), « Mesurer le bénévolat pour en améliorer la connaissance et satisfaire à une recommandation internationale », RECMA - Revue internationale d'économie sociale, no 314, pp. 84-104.

AustralianBureau of Statistics (2000), Unpaid Work and the Australian Economy, 1997, Cat 4240.0, 1999-2000, Canberra.

Australian Bureau of Statistics (2002), Non-profit Institutions Satellite Account, 1999-2000, Canberra.

Bevant D., Noguès H., Prouteau L. (2001), La mesure du bénévolat organisé, rapport final du LEN-CEBS pour la Délégation interministérielle à l'innovation sociale et à l'économie sociale, Maison des sciences de l'homme Ange Guépin.

Boltanski L., Thévenot L. (1991), De la justification. Les économies de la grandeur, Paris, Gallimard.

Brown E. (1999), "Assessing the Value of Volunteer Activity", Nonprofi t and Voluntary Sector Quarterly, Vol. 28, pp. 3-17.

Caillavet F. (1998), « La production domestique des femmes réduit l'inégalité des revenus familiaux », Économie et Statistique, no 311, pp. 75-89.

CERPHI (2006), La France associative en mouvement, $4 \mathrm{e}$ édition.

Chadeau A. (1985), "Measuring Household Activities : Some International Comparisons", The Review of Income and Wealth, Vol. 31, pp. 237-253.

Chadeau A., Fouquet A. (1981), « Peut-on mesurer le travail domestique », Économie et Statistique, no 136, pp. 29-42.

Davin B., Paraponaris A., Verger P. (2009), « Entre famille et marché : déterminants et coûts monétaires de l'aide formelle et informelle reçue par les personnes âgées en domicile ordinaire », Management et Avenir, no 26, pp. 190-204.

FACK G., LANDais C. (2009), « Les incitations fiscales aux dons sont-elles efficaces ? », Économie et Statistique no 427-428, pp. 101-121.

GADREY J. (2006), « Les conventions de richesse au cœur de la comptabilité nationale. Anciennes et nouvelles controverses », in EYMARD-DuvERnAY F., L'économie des conventions, méthodes et résultats, tome I, Paris, La Découverte, pp. 311-324.

GAdRey J., JANY-CATRICE F. (2007), Les nouveaux indicateurs de richesse, Paris, La Découverte.

GoldSChMidT-CleRmont L. (1976) "Monetary Valuation of Non-Market Productive Time : Methodological Considerations", The Review of Income and Wealth, Vol. 39, pp. 419-433.

Gorz A. (1988), Métamorphose du travail. Quête du sens, Paris, Galilée.

HAWRYLYSHYN O. (1976), "The Value of Household Services : A Survey of Empirical Estimations", The Review of Income and Wealth, 1976, Vol., pp. 101-131.

Hawrylyshyn O. (1977), "Towards a Definition of Non-Market Activities", The Review of Income and Wealth, Vol. 23, pp. 79-96.

Herzog A.R., Morgan J.N. (1992), “Age and Gender Differences in the Value of Productive Activities", Research on Aging, Vol. 14, pp. 169-198.

Hourigan M. (2001), Country Fire Authority: Value of the Volunteer contribution - Version 4, February, consultable sur le site internet : http://knowledgeweb.afac.com.au/ research/hr/documents/Value_of_Volunteers_Hourigan. pdf

IRONMONGER D. (2009), The Economic Value of Volunteering in Western Australia, Government of Western Australia, Department for Communities, University of Melbourne.

KAMInski P. (2005), Le compte des institutions sans but lucratif en France, rapport de mission, Paris, INSEE.

Keyboard Roundtable (2003), "Is Assigning a Financial Value to Volunteering a Good Idea", e-Volunteerism, Vol. 3 , Issue 2, pp. 1-10.

Langlais J.-L. (2008), Pour un partenariat renouvelé entre l'État et les associations, ministère de la Santé, des Sports, de la Jeunesse et de la Vie associative.

Low N., Butt S., Ellis Paine A., David Smith J. (2007), Helping out : A national survey of volunteering and charitable giving, National Centre for Social Research, The Institute for Volunteering Research.

Mertens S., LefÈbvre M. (2004), « La difficile mesure du travail bénévole dans les institutions sans but lucratif », in Le compte satellite sans but lucratif 2000-2001, Institut des comptes nationaux-Banque nationale de Belgique.

Mook L., Sousa J., Elgie S., Quarter J. (2005), "Accounting for the Value of Volunteer Contributions", Nonprofi tManagement and Leadership, Vol. 15, pp. 401-415.

MorAnge P. (2008), Rapport d'information sur la gouvernance et le fi nanement des structures 
associatives, Assemblée nationale, Commission des affaires culturelles, familiales et sociales.

Neyret G., Nivlet J.-M., Rault D. (1998), «Associations régies par la loi de $1901 »$, Rapport du CNIS, no 44.

Osberg L., Sharpe S. (1998), An Index of Economic Wellbeing for Canada, Conference on the State of Living Standards and the Quality of Life in Canada, Ottawa, October.

Pно Y.H. (2004), Volunteer Output and the National Accounts : An Empirical Analysis, communication présentée à l'assemblée annuelle de l'Eastern Economic Association, février, disponible sur le site : http://www. bea.gov/papers/pdf/Volunteer_output_paper.pdf

Prouteau L., WolfF F.-C. (2004a), « Donner son temps : les bénévoles dans la vie associative », Économie et Statistique, no 372, pp. 3-39.

Prouteau L., Wolff F.-C. (2004b), « Le travail bénévole : un essai de quantification et de valorisation », Économie et Statistique, no 373, pp. 33-56.

Prouteau L., WolfF F.-C. (2007), « La participation associative et le bénévolat des seniors », Retraite et Société, no 50, pp. 157-189.

Schmid H., Souza-Posa A., Widmer R. (2002), Évaluation monétaire du travail non rémunéré. Une analyse empirique pour la Suisse basée sur l'enquête suisse sur la population active, Neuchâtel, Office fédéral de la statistique.

Simonet-Cusset M. (2004), « Penser le bénévolat comme travail pour repenser la sociologie du travail », Revue de l'IRES, no 44, pp. 141-155.

Statistics New Zealand (2007), Non-profit Institutions Satellite Account : 2004, Wellington.
StatistiqueCanada(2006), Canadiensdévoués, Canadiens engagés. Points saillant de l'enquête canadienne sur le don, le bénévolat et la participation, Ottawa.

Statistique Canada (2008), Comptes satellites des institutions sans but lucratif et du bénévolat. 1997 à 2005, Ottawa.

Steinberg K.S., Rooney P.M., Chin W. (2002), "Measurement of Volunteering : A Methodological Study Using Indiana as a Test Case", Nonprofit and Voluntary Sector Quarterly, Vol. 31, pp. 484-501.

Stiglitz J.-E., Sen A., Fitoussi J.-P. (2009), Rapport de la Commission sur la mesure des performances économiques et $d u$ progrès social, ministère de l'Économie, de l'Industrie et de l'Emploi, La Documentation française.

Talbert J., Cobb C., Slattery N. (2007), The Genuine Progress Indicator 2006. A Tool for Sustainable Development, Redefining progress, 1904 Franklin St., Suite 600, Oakland, CA 4612, USA.

TCHERNONOG V. (2000), Logiques associatives et financement du secteur associatif. Éléments de cadrage, Rapport au ministère de l'Emploi et de la Solidarité, Matisse-LES, université de Paris-I.

TCHERNONOG V. (2007), Le paysage associatif français, Mesures et évolutions, Juris Associations, Dalloz.

United Nations (2003), Handbook on Non-Profit Institutions in the System of National Accounts.

VAn den Berg B., Brouwer W.B.F., Koopmanschap M.A. (2004), "Economic valuation of informal care", European Journal of Health Economics, Vol. 5, pp. 36-45.

VAnoli A. (2002), Une histoire de la comptabilité nationale, Paris, La Découverte. 